\title{
Barriers and facilitators to provide quality TIA care in the Veterans Healthcare Administration OPEN
}

Teresa M. Damush, PhD Edward J. Miech, PhD Jason J. Sico, MD Michael S. Phipps, MD Greg Arling, PhD Jared Ferguson, BS Charles Austin, BA Laura Myers, PhD Fitsum Baye, MS Cherie Luckhurst, PhD

Ava B. Keating, BA Eileen Moran, MS Dawn M. Bravata, MD

Correspondence to Dr. Damush: tdamush@iupui.edu

\section{ABSTRACT}

Objective: To identify key barriers and facilitators to the delivery of guideline-based care of patients with TIA in the national Veterans Health Administration (VHA).

Methods: We conducted a cross-sectional, observational study of 70 audiotaped interviews of multidisciplinary clinical staff involved in TIA care at $14 \mathrm{VHA}$ hospitals. We de-identified and analyzed all transcribed interviews. We identified emergent themes and patterns of barriers to providing TIA care and of facilitators applied to overcome these barriers.

Results: Identified barriers to providing timely acute and follow-up TIA care included difficulties accessing brain imaging, a constantly rotating pool of housestaff, lack of care coordination, resource constraints, and inadequate staff education. Key informants revealed that both stroke nurse coordinators and system-level factors facilitated the provision of TIA care. Few facilities had specific TIA protocols. However, stroke nurse coordinators often expanded upon their role to include TIA. They facilitated TIA care by (1) coordinating patient care across services, communicating across service lines, and educating clinical staff about facility policies and evidence-based practices; (2) tracking individual patients from emergency departments to inpatient settings and to discharge for timely follow-up care; (3) providing and referring TIA patients to risk factor management programs; and (4) performing regular audit and feedback of quality performance data. System-level facilitators included clinical service leadership engagement and use of electronic tools for continuous care across services.

Conclusions: The local organization within a health care facility may be targeted to cultivate internal facilitators and a systemic infrastructure to provide evidence-based TIA care. Neurology ${ }^{\circledR}$ 2017;89:2422-2430

\section{GLOSSARY}

ED $=$ emergency department; FTE $=$ full-time equivalent; VAMC $=$ Veterans Administration Medical Centers; VHA = Veterans Health Administration.

TIA is prevalent among adults, with an estimated overall prevalence of $2.3 \%$ of US adults. ${ }^{1-3}$ Recommended elements of care for patients with TIA and ischemic stroke have been welldescribed in guidelines and include timely diagnostic and therapeutic care processes that seek to identify the etiology of cerebrovascular events and provide high-quality vascular risk factor management. ${ }^{4-9}$ These elements span both clinical specialties and health care settings, requiring cross-service coordination of care. Delivery of guideline-concordant TIA care is associated with significantly lower (70\% reduction) risk of recurrent vascular events ${ }^{10}$ and fewer deaths. ${ }^{11}$

Despite this opportunity to reduce vascular risk, variability exists across hospitals in terms of quality of cerebrovascular care. ${ }^{12}$ Therefore, we sought to conduct a formative evaluation of current TIA practices to identify barriers and facilitators to providing high-quality TIA care in

From the VA PRIS-M QUERI Center (T.M.D., E.J.M., J.F., C.A., L.M., D.M.B.), VA HSR\&D Center for Health Information \& Communication Center (CIN 13-416) (T.M.D., E.J.M., J.F., C.A., L.M., C.L., D.M.B.), Roudebush VAMC (T.M.D., E.J.M., J.F., C.A., L.M., C.L., D.M.B.), Indiana University School of Medicine (T.M.D., E.J.M., C.A., F.B., D.M.B.), Regenstrief Institute, Inc. (T.M.D., E.J.M., A.B.K., D.M.B.), Indianapolis, IN; VA Connecticut Healthcare System (J.J.S.), West Haven, CT; Yale University School of Medicine (J.J.S., M.S.P., J.F.), New Haven; University of Maryland School of Medicine (M.S.P.), Baltimore; School of Nursing (G.A.), Purdue University, West Lafayette, IN; and VA Office of Productivity, Efficiency, and Staffing (E.M.), West Haven, CT.

Go to Neurology.org for full disclosures. Funding information and disclosures deemed relevant by the authors, if any, are provided at the end of the article. The Article Processing charge was funded by VA HSRD PRIS-M QUERI Center, Roudebush VAMC, Indianapolis.

This is an open access article distributed under the terms of the Creative Commons Attribution-NonCommercial-NoDerivatives License 4.0 (CC BY-NC-ND), which permits downloading and sharing the work provided it is properly cited. The work cannot be changed in any way or used commercially without permission from the journal. 
the national Veterans Health Administration (VHA), the largest integrated health care system in the United States, across clinical specialties and across settings of care.

METHODS Interview data. We conducted a formative evaluation in a sample of 14 diverse Veterans Administration Medical Centers (VAMC) across the United States between May 2014 and February 2015. The sampled facilities were chosen from among VAMCs having an annual volume of $\geq 25$ patients with a TIA or minor stroke. ${ }^{13}$ We invited facilities to participate directly through the clinical and facility leadership and obtained permission to visit from each facility Medical Center Director. We also provided advance written notification to the local union. Participation was voluntary and individual responses were deidentified. We alternated scheduling across geographic regions to ensure a representative sample of the VHA system facilities. We continued to sample facilities until we reached information saturation. ${ }^{14}$

Standard protocol approvals, registrations, and patient consents. The Indiana University Institutional Review Board and Roudebush VAMC Research and Development Committee approved the research. We obtained written permission to audiotape staff interviews, which were transcribed verbatim.

We purposely sampled clinical services involved in TIA care and snowballed to local referrals. We completed 70 interviews (68 in person and 2 by telephone) with clinical staff involved with TIA care at 14 participating VAMCs. All transcripts were de-identified and imported into an Nvivo10 project file for data coding and analysis.

To develop the qualitative database, we followed team procedures. ${ }^{14}$ Project team members independently read and coded identical transcripts using a common codebook derived from the semi-structured interview guide. Each coded transcript was merged into a single file, and the project team met as a group to review and discuss similarities and differences in the coding selections until a shared understanding of each codebook item had been developed.

For the analyses presented in this article, we began with coded descriptions of 2 overarching codes: "Barriers and Challenges to TIA Care" and "Facilitators of TIA Care." We compared codes

\begin{tabular}{|ll|}
\hline Table $1 \quad \begin{array}{l}\text { Veterans Health Administration (VHA) facility characteristics: Level of } \\
\text { acute stroke center and regional location }\end{array}$ \\
$\begin{array}{l}\text { Site characteristic } \\
\text { Level of stroke self-designation }\end{array}$ \\
Primary stroke center & 8 \\
Limited hours stroke facility & 4 \\
Stroke support center & 2 \\
US geographic region & \\
East & 4 \\
Midwest & 4 \\
Southwest & 2 \\
West & 4 \\
\hline
\end{tabular}

Primary stroke centers are self-designated VHA facilities that offer acute stroke care 24 hours a day/7 days a week. Limited hour stroke facilities self-designated as offering acute stroke care during the hours between 8 AM and 5 PM during the weekdays and transferring acute stroke patients to nearby community stroke centers during off hours and weekends. Stroke support centers transfer acute stroke patients to nearby community stroke centers 24 hours a day/7 days a week. ${ }^{16}$ across sites and among types of providers. We identified emergent themes and patterns and illustrated these with direct quotations.

Facility characteristics data. In addition, we augmented our interview data with VHA administration data from fiscal year 2011. ${ }^{15}$ We obtained staffing full-time equivalent (FTE) in the emergency department (ED), neurology service, and vascular surgery from the VHA Office of Productivity, Efficiency and Staffing.

We obtained stroke center self-designations from the Acute Ischemic Stroke Directive facility declarations to the VHA Offices of Emergency Medicine and Neurology. ${ }^{16,17}$ Group means between facility data with and without a stroke coordinator were compared with $t$ tests and the group proportion comparisons were compared with $\chi^{2}$ tests.

RESULTS Table 1 displays the level of selfdesignated VA stroke centers and the US region of the 14 sampled facilities. The sites included a diverse VAMC sample both in terms of stroke level selfdesignation and geography. The majority of the sites were self-designated Primary or Limited Hours Stroke Centers. ${ }^{16}$

We interviewed 5 staff members on average per facility. These included 15 (20.3\%) neurologists, 8 (10.8\%) emergency medicine physicians, 7 (9.5\%) ophthalmologists, 7 (9.5\%) vascular surgeons, 6 (8.1\%) ambulatory care physicians, 6 (8.1\%) radiologists, $5(6.7 \%)$ stroke nurses, 4 (5.4\%) chiefs of staff, 4 (5.4\%) hospitalists, 4 (5.4\%) quality management nurses, 3 (4.1\%) cardiologists, $2(2.7 \%)$ neurology advanced practitioner nurses, 1 (1.4\%) ED nurse, 1 (1.4\%) rehabilitation therapist, and 1 (1.4\%) pharmacist.

The facility tenure of the respondents included both recent and long-term employment, which ranged from $\leq 1$ year (10\%) to $2-5$ years $(37 \%)$, $6-15$ years $(27 \%)$, or $\geq 16$ years $(27 \%)$.

Facility characteristics. In table 2, we present site level characteristics of our sample of VAMC facilities, including 6 sites with and 8 sites without a stroke nurse coordinator. The stroke nurse coordinators were designated roles included in a stroke team and directed by a neurologist, often allocated in response to the acute stroke directive. ${ }^{17}$ We split facilities by presence of such coordinators as facilities reported they often extended the stroke coordinator role to include TIA care. Facilities without such coordinators reported no organization of TIA care.

The annual volume of patients with TIA varied widely in facilities with both a stroke coordinator (mean 56.7) and without a nurse stroke coordinator (mean $44.6[p=0.28]$ ). The overall proportion of TIA patients admitted, however, was similar for facilities with (mean 63.5\%) and without a stroke coordinator (mean 65.2\%) ( $p=0.59)$. In general, staffing levels for neurology, emergency medicine, and vascular surgery were greater in sites with a stroke 
Table 2 Veterans Health Administration medical center site level characteristics

\begin{tabular}{|c|c|c|c|c|c|c|c|c|}
\hline \multirow[b]{2}{*}{$\begin{array}{l}\text { Stroke nurse } \\
\text { coordinator }^{\mathrm{a}}\end{array}$} & \multirow[b]{2}{*}{$\begin{array}{l}\text { Annual volume } \\
\text { of TIA patients }\end{array}$} & \multirow{2}{*}{$\begin{array}{l}\text { Proportion of TIA } \\
\text { patients who are } \\
\text { admitted }\end{array}$} & \multicolumn{3}{|c|}{ Staffing levels, FTE } & \multirow[b]{2}{*}{$\begin{array}{l}\text { Stroke level } \\
\text { self-designation }\end{array}$} & \multirow[b]{2}{*}{$\begin{array}{l}\text { Active stroke } \\
\text { team }\end{array}$} & \multirow[b]{2}{*}{$\begin{array}{l}\text { Collects } \\
\text { stroke } \\
\text { data }\end{array}$} \\
\hline & & & Neurology & ED & $\begin{array}{l}\text { Vascular } \\
\text { surgery }\end{array}$ & & & \\
\hline \multirow[t]{6}{*}{ Present } & 95 & 78.9 & 6.79 & 6.81 & 0 & PSC & Yes & Yes \\
\hline & 68 & 64.7 & 3.35 & 7.06 & 2.77 & PSC & Yes & No \\
\hline & 42 & 64.3 & 5.04 & 10.32 & 2.5 & PSC & Yes & Yes \\
\hline & 48 & 62.5 & 4.77 & 3.56 & 1.6 & PSC & Yes & Yes \\
\hline & 43 & 58.1 & 1.53 & 7.12 & 0.56 & SS & No & No \\
\hline & 44 & 52.3 & 5.94 & 9.71 & 2.2 & PSC & Yes & Yes \\
\hline Mean & 56.7 & 63.5 & 4.6 & 7.4 & 1.6 & & & \\
\hline \multirow[t]{8}{*}{ Absent } & 66 & 63.6 & 2.27 & 2.88 & 1.15 & $\mathrm{LH}$ & No & No \\
\hline & 63 & 84.1 & 4.15 & 4.73 & 0.96 & LH & No & No \\
\hline & 54 & 68.5 & 2.52 & 5.15 & 1.63 & $\mathrm{LH}$ & No & No \\
\hline & 51 & 52.9 & 0.32 & 6.98 & 1.98 & PSC & No & No \\
\hline & 45 & 71.1 & 2.62 & 0.04 & 1.27 & SS & No & No \\
\hline & 11 & 45.5 & 5.09 & 0.07 & 1.25 & PSC & No & Yes \\
\hline & 39 & 71.8 & 2.32 & 0 & 1.62 & PSC & No & No \\
\hline & 28 & 64.2 & 3.98 & 8.33 & 1.67 & LH & No & No \\
\hline Mean & 44.6 & 65.2 & 2.9 & 3.5 & 1.4 & & & \\
\hline
\end{tabular}

Abbreviations: $\mathrm{ED}=$ emergency department; $\mathrm{FTE}=$ full-time equivalent; $\mathrm{LH}=$ limited hours facility; PSC = primary stroke center; SS = stroke support center.

Data presented are from fiscal year 2011. Site total TIA = total number of TIA patients cared for at the facility in fiscal year 2011 (excluding minor stroke patients).

a Stroke nurse coordinator was or was not reported as on staff prior to facility interview visit.

coordinator compared to sites without a coordinator, suggesting these sites with a coordinator had a greater capacity to provide acute care and vascular surgical services. The mean total FTE per site was 13.6 for those with and 7.9 for sites without a coordinator $(p=0.01)$, where the bulk of the difference was in ED staffing: mean ED FTE 7.4 per site vs 3.5 per site $(p=0.03)$.

In terms of existing structure for quality improvement, 5 of the 6 sites with nurse stroke coordinators had active stroke teams that participated in quality improvement for stroke care. Moreover, 4 of these 5 facilities with stroke nurse coordinators routinely tracked and collected stroke quality data as part of their quality improvement efforts. In contrast, none of 8 sites without a stroke coordinator had an active stroke team and only 1 of the 8 sites collected stroke quality data at the time of the interview. Respondents reported that they routinely included TIA patients in the quality audits for acute care processes, which overlapped with stroke care although they were not mandated to do so.

Barriers to providing TIA care and facilitators to overcome reported barriers. Our analysis identified barriers to both providing and improving TIA care across the continuum of care from acute care (table 3 ) to the follow-up care period (table 4) as well as reported barriers to improving the quality of TIA care (table 5).

Several emergent themes arose from facilities with a stroke nurse coordinator. These sites utilized and expanded the role of the stroke nurse coordinator to facilitate quality of care for TIA without a specific TIA protocol or a TIA mandate. They overcame barriers to TIA care by using strategies similar to those that had been successfully deployed for stroke care (tables 3-5). System level facilitators were also identified. For example, meetings between services among service chiefs were conducted to decide upon imaging protocols.

Acute care. During acute TIA care, key barriers for facilities without stroke nurse coordinators were access to timely brain imaging, working with a constantly rotating pool of housestaff who needed to learn the facility's protocols, resource constraints related to personnel and services provided, poor care coordination across services, and limited staff and patient education (table 3). Imaging resource constraints also occurred at facilities with and without stroke nurse coordinators. Stroke nurse coordinators facilitated TIA care by coordinating care spanning across services, communicating across service lines, and educating staff. System-level facilitators involved the respective service chiefs' meetings to decide upon protocols for specific processes of care that applied 
Table 3 Barriers and facilitators to providing acute TIA care in the Veterans Health Administration across settings

\section{Quality issue \\ Brain imaging access \\ Barrier}

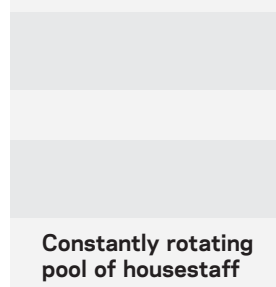

pool of housestaff

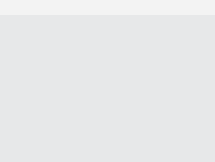

Resource constraints: Personnel

Staff education

Patient factors

No funding for RN or stroke coordinator.wo
Teleradiology is not able to give quick reads. ${ }^{\text {wo }}$

May need to admit patient in order to obtain all tests/studies. ${ }^{\text {Wo,RN }}$

"We are a little behind but within 30 days for vascular studies as well as MRI." [102_400] Radiology ${ }^{\mathrm{RN}}$

Rotating residents have to be constantly educated on what is the appropriate radiology examination to use. ${ }^{\text {wo }}$

"[First year radiology] residents...start as a clean slate...they slow you down, because you really have to teach them and get them up to speed

and so they don't add anything to the productivity to the department." [108_100] Radiologywo
No stroke neurologist on staff.wo

"We only have so many resources so we can't keep activating a stroke code on everybody that comes in." [101_400] Emergency wo

Disconnect between services-between ED and inpatient medicine and neurology. WO, RN

"ED culture is such that they are in a different domain than rest of facility ... At first it was difficult for the ED to think they need to work as a team with other services outside of ED. They also have different bodies of evidence that they follow." [107_200] Nursing

Care coordination between inpatient to discharge. wo

"No specific protocol being followed [by Hospitalists] so things get dropped especially at discharge depending on how meticulous discharge orders are." [103_200] Hospitalists

Provider/staff recognition of TIAs. Wo, RN

Need to reeducate the moonlighters in the ED.wo

"Biggest issue is PC needs to understand what TIA symptoms are and then understand that it's an expedited work up." [106_300] Vascular surgery ${ }^{\mathrm{RN}}$

Patients may wait until the next scheduled appointment to come into the facility. wo

\section{Facilitator to overcome barrier}

Neuroradiologist and chief of neurology meet to discuss and decide use of imaging for the facility. ${ }^{S}$

"We are going to bring that point to the service chief and see if they would be interested." [111_800] Radiology

Stroke RN oversees the acute providers: "I do think this is maybe a little bit unique in that I'm Istroke nursel basically on call 24/7 here. I mean the only times I don't answer my pager is-and even when I'm physically out of town I still answer my pager, so there's just one guy they always know is here, and I have a team...so everyone knows who we are." [113_300] Nursing ${ }^{c}$
Stroke Committee communicates resource needs to facility: "They have been very supportive and...very pleased with everything that we have been doing and then the last meeting...they were very concerned... about not being able to remain a primary center and requested for something to be done." [112 400] Emergencys

Stroke RN talks to and educates the ED staff/clinicians on TIA care processes. $^{\mathrm{C}}$

"Neurology's interactions with the ER, ICU is in its infancy with the stroke code protocol." [107_200] Nursing

Implementation of electronic tools to facilitate practices and protocols. ${ }^{\mathrm{s}}$

"eConsult template [is available in general] to encourage primary providers to ask for an eConsult rather than a consult itself if they think it's only a question." [108_400] Vascular surgery

TIA treatment in the ED appeared to be provider-dependent. In general, TIAs were admitted. Stroke RN suggested use of ABCD2 tool to determine TIA risk but was met with resistance. Discussed evidence and treatment. ${ }^{C}$

"I meet with them all [staff]. Since I've been in this position I educate them [ED]." [112_500] Nursing ${ }^{\mathrm{C}}$

Stroke coordinators are coordinating patient education about signs and symptoms of stroke/TIA at the community outpatient clinics about the patient needing to get urgent care when recognizing symptoms. ${ }^{C}$

"Because [TIA] is resolved and they [patients] feel fine...they end up leaving [AMA]." [104 200] Neurologywo, RN

Abbreviations: $\mathrm{AMA}=$ against medical advice; $\mathrm{C}=$ stroke nurse coordinator facilitator; $\mathrm{ED}=$ emergency department; $\mathrm{ER}=$ emergency room; ICU $=$ intensive care unit; $\mathrm{RN}=$ barriers reported by facilities with a stroke nurse coordinator; $\mathrm{S}=$ system level facilitator; WO = barriers reported by facilities without a stroke nurse coordinator.

to TIA care, the negotiation for resources with leadership by the stroke committee, and the implementation of electronic tools to facilitate best practices.

Follow-up care. As patients transition from acute services to outpatient follow-up care, care coordination and follow-up of risk factor management are clinical challenges of central importance at most facilities (table 4). Some clinicians from facilities without a stroke nurse fretted over whether the discharged patient would get lost in the system and not obtain adequate follow-up care. While many recognized that 
Table 4 Barriers and facilitators to providing follow-up TIA care in the Veterans Health Administration

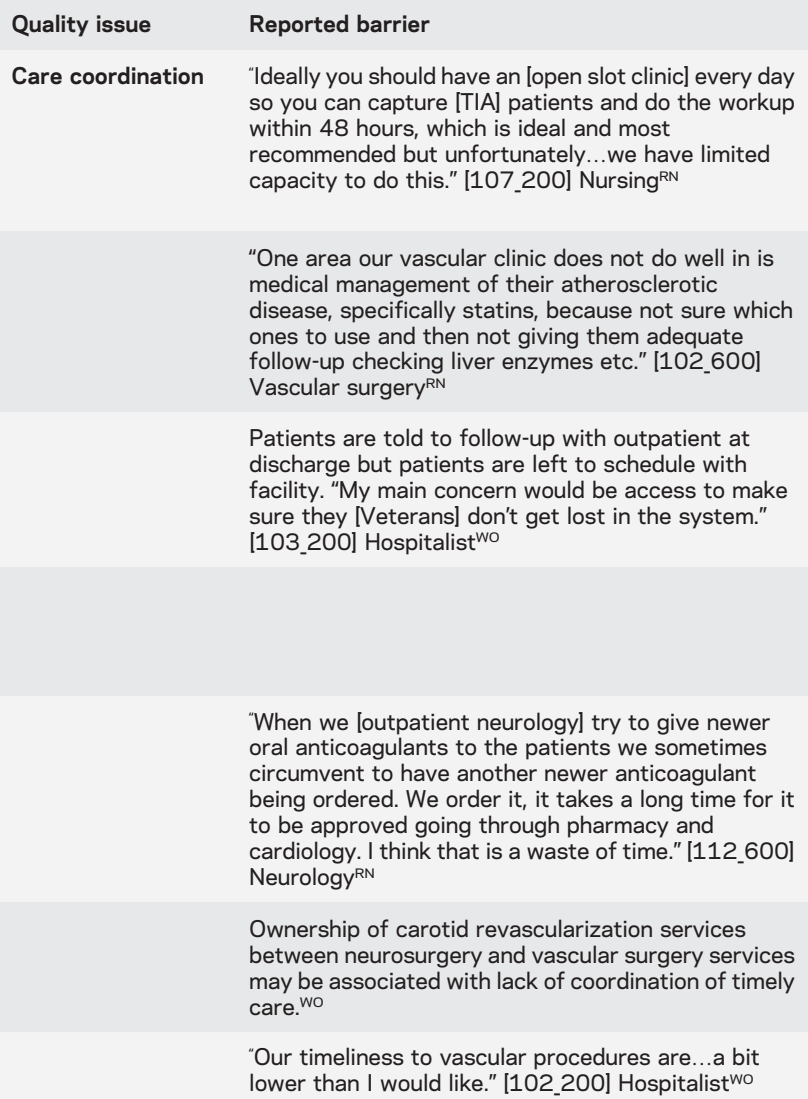

Patients are told to follow-up with outpatient at discharge but patients are left to schedule with facility. "My main concern would be access to make sure they [Veterans] don't get lost in the system." [103_200] Hospitalistwo

"When we [outpatient neurologyl try to give newer oral anticoagulants to the patients we sometimes circumvent to have another newer anticoagulant being ordered. We order it, it takes a long time for it to be approved going through pharmacy and cardiology. I think that is a waste of time." [112 600] Neurology ${ }^{\text {RN }}$

Ownership of carotid revascularization services between neurosurgery and vascular surgery services may be associated with lack of coordination of timely care. wo

"Our timeliness to vascular procedures are.... a bit lower than I would like." [102_200] Hospitalistwo

Facilitator to overcome barrier

"When you meet with [stroke nurse] there's a clinic called the rapid access stroke, TIA clinic....but those are the people who don't qualify necessarily for an acute admission, for those people who need to get an expedited work up and evaluation." [107_300] Neurologyc

Stroke RN follows up with patients at dischargehelps reduce gaps in care. [lf patient's blood pressure is really high] "We're going to have him come back in...to a RN visit to have blood pressure repeated." [102 300] Primary care ${ }^{c}$

Stroke RN follows the TIA patient from inpatient to discharge to follow-up care to ensure receipt of appropriate services as needed. ${ }^{\mathrm{C}}$

"I see the [stroke patient] on the ward...I call [the patient] on the phone...I tell them when they come back to the hospital for another appointment they can come and see me." [112_500] Nursing

Abbreviations: $\mathrm{C}$ = stroke nurse coordinator facilitator; $\mathrm{RN}=$ barriers reported by facilities with a stroke nurse coordinator; $\mathrm{S}=$ system level facilitator; WO = barriers reported by facilities without a stroke nurse coordinator.

timely follow-up care could reduce recurrent risk vascular events, it was often unclear which service would provide secondary preventive care. Sites with stroke nurse coordinators reported assigning them the responsibility of tracking patients from the ED to the inpatient setting and through discharge to the outpatient setting, ensuring that patients received appropriate follow-up care. Stroke nurse coordinators addressed gaps in care by referring the TIA patient to existing preventive risk factor management services and providing secondary risk factor modification education directly to patients. All sites, even those with a nurse resource, identified care coordination across services and patient risk factor management as perceived challenges.

Notably, none of the facilities reported the use of a stroke coordinator to coordinate vascular surgical follow-up for TIA patients. Rather, the clinical staff reported directly placing consults with vascular surgery when needed and assuming that appropriate and timely follow-up would occur without any tracking or confirmatory mechanism. Vascular surgeons reported that once notified of a consult, they provided appropriate and timely TIA care but also did not report the use of any mechanism to identify patients (even a high-risk subset [e.g., those with symptomatic carotid stenosis]) for whom a consult had been placed but who either did not have an appointment scheduled or who missed their appointment.

Improving TIA care quality. The lack of TIA-related data was a commonly reported barrier for facilities without a nurse stroke coordinator (table 5). Neither the clinical nor quality management services had available resources for quality improvement for stroke or TIA care. Sites with stroke nurse coordinators reported collecting and analyzing monthly quality data (both stroke and TIA patients). They fed back the performance quality information to both individuals and groups associated with TIA care even though they were not mandated to track TIA data. Interestingly, data were rarely fed back to vascular surgery.

In addition to the key role of the nurse stroke coordinator, other system-level facilitators that established a climate for implementation of evidence-based practices for TIA care included the following: the facility stroke committee utilizing reporting pathways to executive leadership for resources and process changes; implementation of electronic tools to track patients; templates to communicate across services 
and clinicians, especially between hospitalists and neurologists with primary care physicians and outpatient services; and the reservation of outpatient clinic openings for urgent, at-risk neurologic patients to be seen immediately.

DISCUSSION Our formative evaluation of TIA care in the VHA revealed key organizational barriers to providing guideline concordant TIA care in a coordinated manner across services in a national health care system. Except for stroke nurse coordinators, most respondents discussed TIA care associated with their specific service area only, and often were unaware of any coordination of care across services or settings. Our analyses also demonstrated that some facilities struggled with improving the quality of TIA care. The implications of our findings suggest lost opportunities for future risk modification of cerebrovascular events among patients with TIA given that a TIA is often a harbinger for future cerebrovascular events. ${ }^{4,5}$

In an effort to address these barriers, we found that VAMCs with an existing stroke nurse coordinator within a stroke team reported that they expanded that role to overcome the challenges with the delivery of high-quality TIA care. Key stroke nurse activities included care coordination across services and settings; risk factor modification; educating and training staff; collecting/reporting quality data; following up on problematic issues; and reducing gaps in services, particularly among emergency medicine, inpatient care, and follow-up outpatient services. However, none of the sampled facilities provided care coordination with vascular surgical services specifically for TIA patients or brain imaging coordination with radiology services by stroke coordinators. Rather, clinical staff reported the expectations that individual services

Table 5 Barriers and facilitators to improving TIA care quality

$\begin{array}{ll}\text { Quality issue } & \text { Reported barrier } \\ \text { No TIA quality data } & \text { "Per the VHA directive, I'm not required to keep } \\ \text { track of [TIA] data, so I don't." [101_500] } \\ \text { Nursingwo } \\ \text { "Don't report data yet because it's 0 [cases]." } \\ \text { [103_100] Nursingwo } \\ \text { "I don't think we are data driven." [103_400] } \\ \text { Emergencywo } \\ \text { Insufficient in quality management service } \\ \text { resources to monitor all of our quality } \\ \text { performance measures-difficult to add/devote } \\ \text { to tracking quality measures. Wo }\end{array}$

Facilitator to overcome barrier

Stroke RN reviews quality performance along with stroke team to identify and fix delays/problems.

Stroke RN or an assigned RN tracks and collects stroke quality data per the stroke data. ${ }^{\mathrm{C}}$

Stroke RN or an assigned RN tracks and collects stroke quality data per the stroke data. ${ }^{\mathrm{C}}$

"All of our data is looked at in real time....and on a quarterly and annual basis...so we can see how we're doing for each code stroke or stroke alert and we summarize and evaluate our performance... whatever weaknesses we're having we do our interventions and measure again." [111_900] Nursing/quality

Stroke RN discusses individual quality performance with nursing/resident staff and how to make corrections to improve performance. ${ }^{c}$

Create tension for change: "If you don't recognize your problems and your weaknesses, then how are you ever going to get better? So I'm not afraid to look at those within our department and I'm not afraid to look at them within a facility either, because I think that it's beneficial to look at those things." [108_300] Emergencys

"Little flexibility that an individual (VAMC) facility has to meet their unique individual needs" [108_300] Emergencywo

"I think that there's an attitude of it's not broke, don't fix it." [108_100] Radiologywo

Abbreviations: $\mathrm{C}$ = stroke nurse coordinator facilitator; $\mathrm{RN}$ = barriers reported by facilities with a stroke nurse coordinator; $\mathrm{S}=$ system level facilitator; WO = barriers reported by facilities without a stroke nurse coordinator. 
would indeed conduct follow-up care in a timely manner.

The approach of extending the roles of the stroke team and nurse coordinator to TIA has support in the literature. Recent findings from Canadian Stroke Prevention clinics showed that developing a fast-track system, which includes cross-service collaboration and rapid evaluation, may improve timely carotid endarterectomy in symptomatic patients ${ }^{18}$ and improve stroke prevention efforts with a 24-hour accessible and dedicated TIA clinic. ${ }^{19}$ Thus, a stroke nurse may serve as the facilitator for cross-service collaboration to provide rapid care while the stroke team establishes a seamless transition of timely care to provide TIA guideline practices.

Despite evidence demonstrating the effectiveness of providing timely TIA care and opportunities to improve TIA care quality, there are no external mandates specifically focused on the provision of the delivery of TIA care or TIA quality measurement. For example, neither the Joint Commission nor the American Heart Association/American Stroke Association includes TIA-specific quality measurement programs; rather their measure sets focus on patients with stroke. In response to the VHA National Acute Ischemic Stroke Directive, which mandated that each VA facility declare its organization in 1 of 3 levels of stroke centers, ${ }^{16}$ many VHA stroke centers allocated a nurse specifically to track stroke patients as part of a stroke team. ${ }^{17}$ Although the VHA has implemented performance measurement for patients with stroke, ${ }^{16}$ there are currently no quality measures being tracked in VHA for TIA care evaluation.

In complex care, nurses have been shown to practice as boundary spanners by interacting and navigating across services and settings of their local organization. ${ }^{20}$ Nurses are employed across service areas and settings within a medical facility; however, they report directly to nursing service leadership in VHA. Because of this reporting structure, nurses are part of a unified nursing service, and therefore may not experience as many cross-setting challenges.

In comparison to nursing service, we found emergency medicine physicians less likely to be involved in any of the follow-up care of a TIA patient once that patient was discharged from the ED or was admitted into the medical facility. This ED practice pertained to all diagnoses and not just neurologic conditions. Indeed, communicating, educating, and training ED clinical staff was one of the key tasks a stroke nurse coordinator performed regularly. In addition, the stroke nurses were often full-time staff and assigned to educating, training, and monitoring the constant rotating neurology residents on their TIA processes.

Our findings of networks and communications barriers for TIA care across services and settings in the VHA were similar to those reported in another national health care system ${ }^{21}$ in the United Kingdom. They identified the challenges in coordinating a multitude of clinical processes spanning across services and settings. To improve their quality of TIA care in the United Kingdom, they began with audit and feedback while providing seminars to the practitioners on appropriate evidence-based care. ${ }^{21}$ In a similar manner in VHA, some facilities extended the stroke coordinator's role to also audit the TIA quality data. Often the quality indicators were only those that overlapped with stroke and TIA and did not include TIA-specific indicators.

The primary stroke centers allocated stroke nurse coordinators for stroke/TIA care somewhat more often than other self-designated levels. While this may suggest these facilities had greater resources to warrant such an approach, some facilities chose to reallocate existing nursing FTE into this role rather than new hires. Moreover, depending on structure of neurology services, stroke nurses operated within a stroke team directed by a neurologist. Thus, the development of stroke coordinators may be a worthwhile resource allocation to facilitate quality TIA care.

Most VHA facilities developed a stroke protocol developed in response to the VHA Acute Ischemic Stroke Directive. ${ }^{16}$ However, the system did not include a mandate for TIA care as part of the directive. Therefore only 1 of the 14 facilities had a specific TIA protocol during our study period. Most reported acutely treating TIA patients with their stroke protocol until a stroke diagnosis was ruled out. Similarly, among facilities with a 24-hour access primary stroke center, none of those sites discussed providing 24hour access for TIA care. ${ }^{19}$

We identified important system-level facilitators. Stroke teams were often tasked with making formal recommendations to and negotiations with hospital leadership for resource allocations. In addition, tools to facilitate care coordination of stroke care were often implemented in the VHA electronic health records. ${ }^{17}$ However, unlike the FASTEST trial, which demonstrated significantly greater delivery of guideline-adherent care with the implementation of a TIA/stroke electronic decision support tool, ${ }^{10}$ facilities in our study did not report electronic tools specifically for TIA care.

Our study had several limitations. First, our sample included staff who volunteered to complete interviews. Staff who did not volunteer may hold different perceptions. However, we did sample in the health care system until we reached data saturation. Second, our data were collected cross-sectionally; thus, we could not track changes in TIA care. Third, we were not able to provide respondents with their transcribed interviews to review and edit. Finally, we were not 
able to link the facilitators and barriers directly to care processes or to outcomes for patients in these facilities. Future studies may possibly investigate the effect of the barriers on clinical processes or outcome measures.

Nonetheless, our study is one of the first formative evaluations of the organization and delivery of TIA care in a national health care system demonstrating that a variety of barriers and some facilitators exist in providing high-quality post-TIA care. An understanding of these barriers and facilitators may serve to inform providers and facility administrators about the development and implementation of interventions to improve the delivery of high-quality care in a population at relatively high risk of future vascular events. Our findings support that stroke nurse coordinators within a stroke team, alongside other systemlevel facilitators, may be a worthwhile allocation of facility resources to improve the delivery of quality of TIA care, and ultimately, patient outcomes.

\section{AUTHOR CONTRIBUTIONS}

Dr. Damush: manuscript preparation, manuscript revision, qualitative interviews, data collection, data analysis. Dr. Miech: manuscript revision, qualitative interviews, data collection, data analysis. Dr. Sico: manuscript preparation, manuscript revision, qualitative interviews, data collection, data analysis. Dr. Phipps: qualitative interviews, data collection, data analysis. Dr. Arling: manuscript preparation, manuscript revision, qualitative interviews, data collection, data analysis. J. Ferguson: manuscrip revision, data collection, data analysis. C. Austin: data collection, data analysis, qualitative interviews. Dr. Myers: manuscript revision, data analysis. F. Baye: manuscript revision, data analysis. Dr. Luckhurst: data analysis. A.B. Keating: data analysis. E. Moran: data collection. Dr. Bravata: manuscript preparation, manuscript revision, data collection, data analysis.

\section{ACKNOWLEDGMENT}

The authors thank the clinical staff and respondents from the 14 VA Medical Centers for their time in completing the interviews.

\section{STUDY FUNDING}

Funding was provided by the Veterans Administration Health Services Research and Development QUERI (Quality Enhancement Research Initiative) Service Directed Project awarded to Dr. Bravata (SDP 12-178) and Health Services Research and Development Career Developmen Award for Dr. Jason Sico (HSRD CDA 1 IK2 HX001388-01A1 "Improving Cerebrovascular Risk Factor Management in Post-Stroke Veterans"). The Article Processing Charge was funded by VA HSRD PRIS-M QUERI Center, Roudebush VAMC, Indianapolis, IN.

\section{DISCLOSURE}

T. Damush and E. Miech report no disclosures relevant to the manuscript. J. Sico is funded by a VA Health Services Research and Development Career Development Award grant CDA 11-262 and has served on a medical advisory board for Acorda Therapeutics. M. Phipps, G. Arling, J. Ferguson, and C. Austin report no disclosures relevant to the manuscript. L. Myers has received research funding from the Veterans Health Administration and Genentech. F. Baye, C. Luckhurst, A. Keating, E. Moran, and D. Bravata report no disclosures relevant to the manuscript. Go to Neurology.org for full disclosures.

Received April 21, 2017. Accepted in final form September 19, 2017.

\section{REFERENCES}

1. Kleindorfer D, Panagos P, Pancioloi A, et al. Incidence and short-term prognosis of transient ischemic attack in a population-based study. Stroke 2005;36:720-723.

2. Johnson SC, Fayad PB, Gorelick PB, et al. Prevalence and knowledge of transient ischemic attack among US adults. Neurology 2003;60:1429-1434.

3. Cancelli I, James F, Gigli GL, et al. Incidence of transient ischemic attack and early stroke risk: validation of the ABCD2 score in an Italian population-based study. Stroke 2011;42:2751-2757.

4. Wu CMMK, Lorenzetti DL, Hill MD, Manns BJ, Ghali WA. Early risk of stroke after transient ischemic attack: a systematic review and meta-analysis. Arch Intern Med 2007;167:2417-2422.

5. Hankey GJ. Impact of treatment of people with transient ischemic attack on stroke incidence and public health. Cerebrovas Dis 1996;6(suppl):26-33.

6. Easton J, Saver J, Albers G, et al. Definition and evaluation of transient ischemic attack. Stroke 2009;40: 2276-2293.

7. Kernan WN, Ovbiagele B, Black HR, et al. Guidelines for the prevention of stroke in patients with stroke and transient ischemic attack: a statement for healthcare professionals from the American Heart Association/American Stroke Association Council on Stroke. Stroke 2014;45: 2160-2236.

8. Sacco R, Adams R, Albers G, et al. Guidelines for prevention of stroke in patients with ischemic stroke or transient ischemic attack. Circulation 2006;113: e409-e449.

9. Adams HPAR, Brott T, del Zoppo GJ, et al. Guidelines for the early management of patients with ischemic stroke. Stroke 2003;34:1056-1083.

10. Rothwell PM, Giles MF, Chandratheva A, et al. Effect of urgent treatment of transient ischaemic attack and minor stroke on early recurrent stroke (EXPRESS study): a prospective population-based sequential comparison. Lancet 2007;370:1432-1442.

11. Ranta A, Dovey S, Weatherall M, O'Dea D, Gommans J, Tilyard M. Cluster randomized controlled trial of TIA electronic decision support in primary care. Neurology 2015;84:1545-1551.

12. O'Brien E, Zhao X, Fonarow G, et al. Quality of care and ischemic stroke risk after hospitalization for transient ischemic attack: findings from Get with the Guidelines-Stroke. Circ Cardiovasc Qual Outcomes 2015;8: S1117-S124

13. Strambo D, Zambon A, Roveri L, et al. Defining minor symptoms in acute ischemic stroke. Cerebrovasc Dis 2015; 39:209-215.

14. Miles MB, Huberman AM, Saldana J. Qualitative Data Analysis: A Methods Sourcebook. Los Angeles, CA: Sage Publications; 2014.

15. Bravata DM, Myers L, Cheng E, et al. Quality of care for veterans with TIA and minor stroke. Stroke. 2015;46 (suppl 1):ATMP73.

16. Department of Veterans Affairs. VHA Directive 2011-038. Treatment of Acute Ischemic Stroke (AIS). Washington, DC: Veterans Health Administration; 2011.

17. Damush TM, Miller KK, Plue L, et al. National implementation of acute stroke care centers in the Veterans Health Administration (VHA): formative evaluation of the field response. J Gen Intern Med 2014;29(suppl 4): $845-852$. 
18. Gocan S, Bourgoin A, Blacquiere D, Shamloul R, Dowlatshashi D, Stotts G. Fast-Track systems improve timely carotid endarterectomy in stroke prevention outpatients. Can J Neurol Sci 2016;43:648-654.

19. Lavellee PC, Meseguer E, Abboud H, et al. A transient ischaemic attack clinic with round-the-clock access (SOS-TIA): feasibility and effects. Lancet Neurol 2007; 6:953-960.
20. Abrahamson K, Mueller C, Davila HW, Arling G. Nurses as boundary-spanners in reducing avoidable hospitalizations among nursing home residents. Res Gerontol Nurs 2014;7:235-243.

21. Wright J, Harrison S, McGeorge M, et al. Improving the management and referral of patients with transient ischaemic attacks: a change strategy for a health community. Qual Saf Health Care 2006;15:9-12.

\section{Get Connected. Stay Connected.}

Connect with the American Academy of Neurology's popular social media channels to stay up-todate on the latest news and breakthroughs in neurology, and network with peers and neurology thought leaders. Visit AAN.com/Connect.

\section{Improve Your Patients' Experience with Free Electronic Exam Room Poster!}

Health Monitor Network is offering electronic touchscreen posters-featuring interactive educational charts, diagrams, and tips on managing neurologic conditions-free to US members for their exam rooms. Improve your patients' experience by ordering yours today: Healthmonitor.com/products/aan-dep/

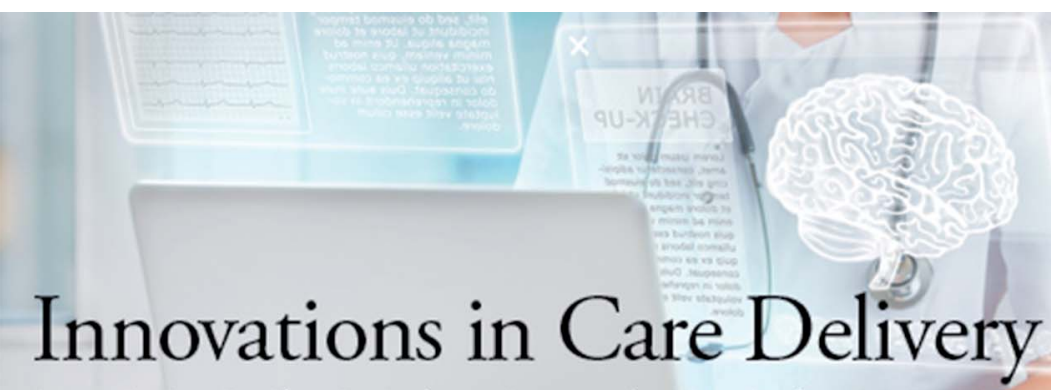

A curated collection featuring advances in neurologic care

\section{Innovations in Care Delivery - A curated collection featuring advances in neurologic care}

This Neurology ${ }^{\circledR}$ special interest website provides a forum to explore new care models from multiple disciplines, access to sources on health care innovation, and expert opinions on current research from Neurology journals. Curated by Brian C. Callaghan, MD, and Kevin A. Kerber, MD.

Stay ahead of the curve at Neurology.org/innovations. 


\section{Neurology}

\section{Barriers and facilitators to provide quality TIA care in the Veterans Healthcare Administration}

Teresa M. Damush, Edward J. Miech, Jason J. Sico, et al.

Neurology 2017;89;2422-2430 Published Online before print November 8, 2017

DOI 10.1212/WNL.0000000000004739

This information is current as of November 8, 2017

\section{Updated Information \&} Services

References

Subspecialty Collections

Permissions \& Licensing

Reprints including high resolution figures, can be found at: http://n.neurology.org/content/89/24/2422.full

This article cites 19 articles, 8 of which you can access for free at: http://n.neurology.org/content/89/24/2422.full\#ref-list-1

This article, along with others on similar topics, appears in the following collection(s):

\section{All Cerebrovascular disease/Stroke}

http://n.neurology.org/cgi/collection/all_cerebrovascular_disease_strok e

All Health Services Research

http://n.neurology.org/cgi/collection/all_health_services_research Stroke prevention

http://n.neurology.org/cgi/collection/stroke_prevention

Information about reproducing this article in parts (figures,tables) or in its entirety can be found online at:

http://www.neurology.org/about/about_the_journal\#permissions

Information about ordering reprints can be found online:

http://n.neurology.org/subscribers/advertise

Neurology ${ }^{\circledR}$ is the official journal of the American Academy of Neurology. Published continuously since 1951, it is now a weekly with 48 issues per year. Copyright Copyright (C) 2017 The Author(s). Published by Wolters Kluwer Health, Inc. on behalf of the American Academy of Neurology.. All rights reserved. Print ISSN: 0028-3878. Online ISSN: 1526-632X.

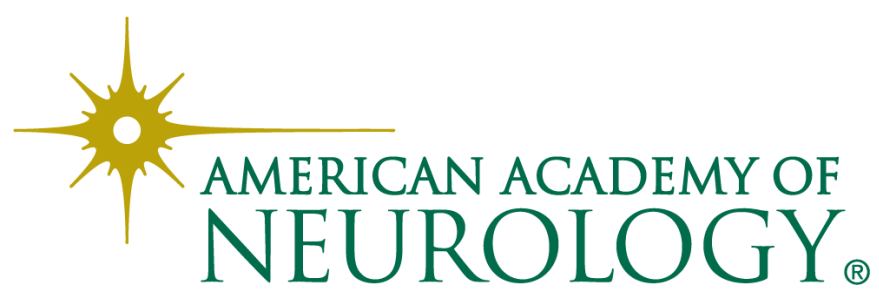

